\title{
Ground-state properties of a supersolid in RPA
}

\author{
A.J. Stoffel ${ }^{1,2}$ and M. Gulácsi ${ }^{1,2, a}$ \\ 1 Max-Planck-Institute for the Physics of Complex Systems, 01187 Dresden, Germany \\ ${ }^{2}$ Nonlinear Physics Centre, Australian National University, Canberra, ACT 0200, Australia
}

Received 16 January 2009

Published online 3 March 2009 - (c) EDP Sciences, Società Italiana di Fisica, Springer-Verlag 2009

\begin{abstract}
We investigate the newly discovered supersolid phase by solving in random-phase approximation the anisotropic Heisenberg model of the hard-core boson ${ }^{4} \mathrm{He}$ lattice at zero temperature. We include nearest and next-nearest neighbor interactions and calculate exactly all pair correlation functions in a cumulant decoupling scheme. We demonstrate the importance of vacancies and interstitials in the formation of the supersolid phase. The supersolid phase is characterised by strong quantum fluctuations which are taken into account rigorously. Furthermore we confirm that the superfluid to supersolid transition is triggered by a collapsing roton minimum however is stable against spontaneously induced superflow, i.e. vortex creation.
\end{abstract}

PACS. 05.30.Jp Boson systems - 67.80.-s Quantum solids - 67.80.bd Superfluidity in solid ${ }^{4}$ He, supersolid ${ }^{4} \mathrm{He}-75.10 . J m$ Quantized spin models

\section{Introduction}

The counterintuitive idea of a superflow in a solid, later coined supersolidity was firstly conjectured in 1969 by Andreev [1] and in 1970 seized by Leggett and Chester $[2,3]$. From a theoretical point of view, supersolidity is a state of matter characterised by simultaneous off-diagonal (ODLRO) and diagonal long range order (DLRO). It was speculated that such a phase exists because vacancies and other defectons are non-localised and will Bose condense at sufficiently low temperature. Still most physicists remained critical of the notion as several experiments failed to produce any evidence of this state. Finally in $2004 \mathrm{Kim}$ and Chan [4,5] measured a tiny superflow in solid helium at temperatures below $T=0.2 \mathrm{~K}$, expressed by non-classical rotational inertia in a torsional oscillation experiment, and thus proved the existence of the supersolid state. This landmark experiment rekindled vast interest in the supersolid state and subsequently many new theories and numerical quantum Monte-Carlo calculations supporting the existence of supersolidity were proposed. However the true nature of the supersolid phase still remains obscure. Numerous follow-up experiments managed to shed light on the matter but the relevance of ${ }^{3} \mathrm{He}$ impurities and especially the nature of the unconventional normal solid to supersolid transition resembling the 2D Kosterlitz-Thouless transition is still being debated.

Recent experiments [6] raised new questions as it was found that the supersolid phase exhibits a hysteresis, where the superfluid signal depends on the chronology of variation of temperature and in the amplitude of the

\footnotetext{
a e-mail: miklos.gulacsi@anu.edu.au
}

rotational oscillation. An other recent experiment [7] detected a change in the elastic properties of solid helium. The change of the elastic moduli bears a remarkable resemblance with the supersolid signal.

However, despite sophisticated numerical methods and advanced theories such as vortex liquids [8] and superglass states [9] we believe that there remains a gap in the range of theories of the supersolid phase. In this paper we intend to fill this gap and present a theory of supersolidity in a quantum lattice gas (QLG) model beyond classical mean-field. We follow the approach of Liu and Fisher [10] and map the QLG model to the anisotropic Heisenberg model. The method of Green's functions proved to be very successful in the description of ferromagnetic and antiferromagnetic states and we use this method to investigate the supersolid phase which corresponds to a canted anti-ferromagnetic phase.

The emergent third order Green's functions in the random-phase approximation (RPA) are broken down using the cumulant decoupling to yield a closed set of equations. Quantum fluctuations at zero temperature result in vacancies and interstitials present even at zero temperature and in the supersolid phase the net vacancy density is therefore non zero. The supersolid phase is characterized by Bose condensation of the vacancies as well as the interstitials and thus both will contribute to superfluidity. Interestingly, the major contribution comes from vacancies. Also, our model confirms propositions that the superfluid to supersolid transition is triggered by a collapsing roton minimum $[11,12]$. Nonetheless our solution shows, contrary to earlier results that this transition is stable against spontaneously induced superflow. 
The paper is organized as follows: in Sections 2 and 3 we introduce the generic Hamiltonian of a bosonic many body system and discretize it to a quantum lattice gas model. This model is equivalent to the anisotropic Heisenberg model in an external field and we will identify the corresponding phases. In the following two sections we derive basic thermodynamic properties relevant at zero temperature and discuss the excitation spectrum of the spin waves in the superfluid and the supersolid phases. The relevance of quantum fluctuations is discussed in Section 7, where we provide justification of results briefly reported elsewhere [13]. Finally in the last two sections we discuss key properties of the supersolid phase and their occurrence within the example of three different sets of coupling constants.

\section{Generic Hamiltonian}

Apart from possible ${ }^{3} \mathrm{He}$ impurities the supersolid Helium-4 state is a bosonic system and the generic Hamiltonian for such systems in the language of second quantisation is given by:

$$
\begin{aligned}
H= & \int d^{3} x \psi^{\dagger}(x)\left(-\frac{1}{2 m} \nabla^{2}+\mu\right) \psi(x) \\
& +\frac{1}{2} \int d^{3} x d^{3} x^{\prime} \psi^{\dagger}(x) \psi^{\dagger}\left(x^{\prime}\right) V\left(x-x^{\prime}\right) \psi(x) \psi\left(x^{\prime}\right)
\end{aligned}
$$

where $\psi^{\dagger}(x)$, the particle creation operator and $\psi^{\dagger}(x)$, the corresponding destruction operator obey the usual bosonic commutator relations. Hamiltonians in three dimensions such as in equation (1) are not solvable even for elementary potentials $V(x)$ such as the Dirac delta distribution. Therefore we are induced to introduce further approximations. An approximation which proved particularly successful for the description of liquid Helium is know as the Quantum Lattice Gas model and was first introduced by Matsubara and Matsuda [14].

In the quantum lattice gas model one works with a space lattice of discrete lattice points rather than the continuum. This approximation shows to be very useful for the supersolid state as the spacial discretization of the this model serves as a natural frame for the crystal lattice of (super)-solid helium. This procedure significantly simplifies the problem of breaking translational invariance symmetry for states that exhibit diagonal long range order. In this way this model gives the easiest possible access to analyze states that exhibit both diagonal and off diagonal long range order simultaneously. Also in this model no specific knowledge of the density distribution of the atoms is needed.

According to Matsubara and Tsuneto [15] the generic Hamiltonian equation (1) in the discrete lattice model reads:

$$
H=\mu \sum_{i} n_{i}+\sum_{i j} u_{i j}\left(a_{i}^{\dagger}-a_{j}^{\dagger}\right)\left(a_{i}-a_{j}\right)+\sum_{i j} V_{i j} n_{i} n_{j}
$$

here $u_{i j}$ are non-zero for nearest neighbor and next nearest neighbor hopping and otherwise zero. The values of $u_{n n}$ and $u_{n n n}$ are such that the kinetic energy is isotropic up to the 4th order. In the case of a BCC lattice (two interpenetrating $\mathrm{SC}$ lattices) the matrix elements are given by:

$$
\begin{aligned}
u_{n n} & =\frac{2}{3} \frac{1}{4 m a^{2}} \\
u_{n n n} & =\frac{1}{3} \frac{1}{4 m a^{2}} .
\end{aligned}
$$

As atoms do not penetrate each other there can exist only one atom at a time on a lattice site and consequently $a^{\dagger}$ and $a$ are the creation and annihilation operators of a hard core boson commuting on different lattice sites:

$$
\left[a_{i}^{\dagger}, a_{j}^{\dagger}\right]_{-}=\left[a_{i}, a_{j}\right]_{-}=\left[a_{i}, a_{j}^{\dagger}\right]_{-}=0(i \neq j)
$$

but obey the anti-commutator relations on identical sites:

$$
\begin{array}{r}
{\left[a_{i}^{\dagger}, a_{i}^{\dagger}\right]_{+}=\left[a_{i}, a_{i}\right]_{+}=0} \\
{\left[a_{i}, a_{i}^{\dagger}\right]_{+}=1 .}
\end{array}
$$

Equation (2) is the Hubbard model in 3 dimensions for hard core bosons. Due to the unusual statistics of hard core bosons, there does not exist a Wick's theorem for their operators and the common formalism of pertubative field theory is not applicable. Hence in the following chapter we transform the model to an equivalent spin model namely the anisotropic Heisenberg model.

\section{Anisotropic Heisenberg model}

It is well known that the operators of hard-core bosons obey the same $\mathrm{SU}(2)$ algebra as spin $S=1 / 2$ particles do. Therefore it is feasible to replace the creation and annihilation operators by spin operators.

$$
\begin{aligned}
& a_{j}^{\dagger}=S_{j}^{x}-i S_{j}^{y} \\
& a_{j}=S_{j}^{x}+i S_{j}^{y} \\
& n_{j}=\frac{1}{2}-S_{j}^{z} .
\end{aligned}
$$

It is easily apprehensible that the usual lie algebra for spin $1 / 2$ particles preserves the mixed commutation/anticommutation relations for hard-core bosons. This substitution transforms the hard-core bosonic Hubbard model into a spin model:

$$
\begin{aligned}
H= & \mu \sum_{i}\left(\frac{1}{2}-S_{i}^{z}\right) \\
& +\sum_{i j} u_{i j}\left(1-S_{i}^{z}-S_{j}^{z}-2 S_{i}^{x} S_{j}^{x}-2 S_{i}^{y} S_{j}^{y}\right) \\
& +\sum_{i j} V_{i j}\left(\frac{1}{4}-\frac{S_{i}^{z}}{2}-\frac{S_{j}^{z}}{2}+S_{i}^{z} S_{j}^{z}\right) .
\end{aligned}
$$




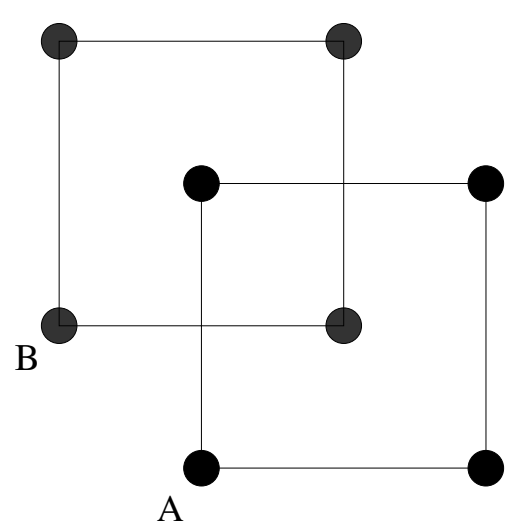

Fig. 1. The BCC lattice consists of two interpenetrating SC sub-lattices. In the perfect solid phase one sub-lattice (i.e. sublattice A) serves as a lattice of on-site centers and is occupied while sub-lattice B represents the empty interstitial and is vacant.

If we adjust the notation to conform with the usual standards of spin models, it becomes evident that the resulting Hamiltonian is the anisotropic Heisenberg model :

$$
\begin{aligned}
H= & -h^{z} \sum_{i} S_{i}^{z}-\sum_{i j} J_{i j}^{\|} S_{i}^{z} S_{j}^{z} \\
& -\sum_{i j} J_{i j}^{\top}\left(S_{i}^{x} S_{j}^{x}+S_{i}^{y} S_{j}^{y}\right)
\end{aligned}
$$

with:

$$
\begin{aligned}
J_{i j}^{\|} & =-V_{i j} \\
J_{i j}^{\top} & =2 u_{i j} \\
h^{z} & =\mu-\sum_{j} J_{i j}^{\top}+\sum_{j} J_{i j}^{\|} .
\end{aligned}
$$

The Hubbard model as well as the anisotropic Heisenberg model are defined on a discrete lattice and one may ask to what extend a specific choice of lattice geometry will affect the physical properties of the system. While the quantitative results certainly depend on the lattice geometry we can safely assume that qualitative properties, such as phase transitions and critical constants will not change for different lattices as long as no frustration effects are evoked. Therefore we may safely chose, in order to avoid unnecessary complications, a simple lattice geometry and an obvious choice are two interpenetrating simple cubic sub-lattices which together form a BCC lattice, see Figure 1. Defining two sub-lattices gives us the possibility to establish the DLRO of solids in a natural way: sublattice A represents the centers of the ${ }^{4} \mathrm{He}$ ions, hence it coincides with the ion lattice. Sub-lattice B defines the interstitial, the space in-between those atomic centers. In the liquid phases of course the occupation number on both sub-lattices is equal as there is no spacial density variation. Table 1 charts the the various magnetic phases and identifies the corresponding phases of the ${ }^{4} \mathrm{He}$ system. According to the spin configurations we call the four magnetic phases ferromagnetic, canted ferromagnetic, canted
Table 1. Possible magnetic phases and the corresponding phases of the Hubbard model. All Phases are defined by their long range order. The columns, from left to right, are the spin configurations, magnetic phases, ODLRO, DLRO and corresponding ${ }^{4} \mathrm{He}$ phases, respectively.

\begin{tabular}{ccccc}
\hline$\uparrow \uparrow$ & FE & No & No & Normal liquid \\
$\nearrow \nearrow$ & CFE & Yes & No & Superfluid \\
$\nearrow \swarrow$ & CAF & Yes & Yes & Supersolid \\
$\uparrow \downarrow$ & $\mathrm{AF}$ & No & Yes & Normal solid \\
\hline
\end{tabular}

anti-ferromagnetic and anti-ferromagnetic phases which we abbreviate by FE, CFE, CAF and AF. The order parameters, $m_{1}$ for off-diagonal long range order and $m_{2}$ for diagonal long range order in the magnetic system are defined by:

$$
\begin{aligned}
& m_{1}=\left\langle S_{A}^{x}\right\rangle+\left\langle S_{B}^{x}\right\rangle \\
& m_{2}=\left\langle S_{A}^{z}\right\rangle-\left\langle S_{B}^{z}\right\rangle .
\end{aligned}
$$

In the following we will use these order parameters to identify the phases within the classical mean-field approximation as was derived by Matsuda and Tsuneto [15] and extended to finite temperature by Fisher and Liu [10] as well as in the novel random-phase approximation.

\section{Green's function}

The anisotropic Heisenberg model in an external field is, not least due to absence of $\mathrm{O}(3)$ symmetry difficult to solve. However, in the context of supersolidity, investigating the CAF phase, it has been solved in a classical meanfield approximation $[10,15]$. The anisotropic Heisenberg Hamiltonian in the classical mean-field approximation is obtained by substituting the spin- $1 / 2$ operators with their respective expectation values:

$$
\begin{aligned}
H_{M F}= & -h^{z}\left(\left\langle S_{A}^{z}\right\rangle+\left\langle S_{B}^{z}\right\rangle\right)-2 J_{1}^{\|}\left\langle S_{A}^{z}\right\rangle\left\langle S_{B}^{z}\right\rangle \\
& -J_{2}^{\|}\left(\left\langle S_{A}^{z}\right\rangle\left\langle S_{A}^{z}\right\rangle+\left\langle S_{B}^{z}\right\rangle\left\langle S_{B}^{z}\right\rangle\right) \\
& \left.-2 J_{1}^{\top}\left\langle S_{A}^{x}\right\rangle\left\langle S_{B}^{x}\right\rangle-J_{2}^{\top}\left(\left\langle S_{A}^{x}\right\rangle\left\langle S_{A}^{x}\right\rangle+\left\langle S_{B}^{x}\right\rangle S_{B}^{x}\right\rangle\right)
\end{aligned}
$$

where $J_{1}^{\|}=q_{1} J_{i \in A j \in B}^{\|}, J_{2}^{\|}=q_{2} J_{i \in A j \in A}^{\|}, J_{1}^{\top}=q_{1} J_{i \in A j \in B}^{\top}$ and $J_{2}^{\top}=q_{1} J_{i \in A j \in A}^{\top}, q_{1}=6$ and $q_{2}=8$ are the number of nearest and next nearest neighbors. Although the classical mean-field theory is quite insightful and gives an accurate description of the variously ordered phases its fails to take quantum fluctuations and quasi-particle excitations into account. Hence, in order to overcome these shortcomings we derive a fully quantum mechanical approximation and solve the anisotropic Heisenberg model in the randomphase approximation which is based on the Green's function technique. At zero temperature the retarded and 
advanced Tyablikov [17,18] commutator Green's function defined in real time are:

$$
\begin{aligned}
& G_{i j_{\text {Ret }}}^{\mu \nu}(t)=-i \theta(t)\left\langle n_{0}\left|\left[S_{i}^{\mu}(t), S_{j}^{\nu}\right]\right| n_{0}\right\rangle \\
& G_{i j_{\text {Adv }}}^{\mu \nu}(t)=i \theta(-t)\left\langle n_{0}\left|\left[S_{i}^{\mu}(t), S_{j}^{\nu}\right]\right| n_{0}\right\rangle
\end{aligned}
$$

here $\left|n_{0}\right\rangle$ is the normalized Heisenberg ground state, $\mu$ and $\nu$ are elements of $\{x, y, z\}$ and $i$ and $j$ denote the lattices sites. The most successful technique of solving many body Green's function involves the method of equation of motion which is given by:

$$
\begin{aligned}
i \partial_{t} G_{i j_{\text {Ret }}}^{x y}(t) & =\delta(t)\left\langle\left[S_{i}^{x}, S_{j}^{y}\right]\right\rangle-i \theta(t)\left\langle\left[\left[S_{i}^{x}, H\right], S_{j}^{y}\right]\right\rangle \\
i \partial_{t} G_{i j_{A d v}}^{x y}(t) & =\delta(t)\left\langle\left[S_{i}^{x}, S_{j}^{y}\right]\right\rangle+i \theta(-t)\left\langle\left[\left[S_{i}^{x}, H\right], S_{j}^{y}\right]\right\rangle .
\end{aligned}
$$

The commutator $\left[S_{i}^{x}, H\right]$ can be eliminated by using the Heisenberg equation of motion giving rise to higher, third order Green's functions on the RHS. In order to obtain a closed set of equations we apply cumulant decoupling procedure and as a consequence the third order Green's functions split into product terms of single operator expectation values and second order Green's functions. The cumulant decoupling [20] is based on the assumption that the last term of the following equality is negligible:

$$
\begin{aligned}
\langle\hat{A} \hat{B} \hat{C}\rangle= & \langle\hat{A}\rangle\langle\hat{B} \hat{C}\rangle+\langle\hat{B}\rangle\langle\hat{A} \hat{C}\rangle \\
& +\langle\hat{C}\rangle\langle\hat{A} \hat{B}\rangle-2\langle\hat{A}\rangle\langle\hat{B}\rangle\langle\hat{C}\rangle \\
& +\langle(\hat{A}-\langle\hat{A}\rangle)(\hat{B}-\langle\hat{B}\rangle)(\hat{C}-\langle\hat{C}\rangle)\rangle .
\end{aligned}
$$

This decoupling scheme leads to a closed set of six equations that determine the six Green's functions, corresponding to three spin components on two sub-lattices.

The cumulant decoupling sheme introduces meanfields of the spins operators which have to be calculated in a self-consistent manner. The Green's functions gives us the possibility to calculate a set of two self-consistency equations. However, in the supersolid and superfluid case the off-diagonal long range order; i.e. non-zero transversal fields $\left\langle S_{A}^{x}\right\rangle$ and $\left\langle S_{A}^{x}\right\rangle$ increase the degrees of freedom in number by two and therefore analytical properties of the commutator Green's functions pose two additional constraints on the spin-fields given by:

$$
\begin{aligned}
& h^{z}+2\left\langle S_{A}^{z}\right\rangle\left(J_{2}^{\|}-J_{2}^{\top}\right)+2\left\langle S_{B}^{z}\right\rangle J_{1}^{\|}=2 J_{1}^{\top} \frac{\left\langle S_{B}^{x}\right\rangle}{\left\langle S_{A}^{x}\right\rangle}\left\langle S_{A}^{z}\right\rangle \\
& h^{z}+2\left\langle S_{B}^{z}\right\rangle\left(J_{2}^{\|}-J_{2}^{\top}\right)+2\left\langle S_{A}^{z}\right\rangle J_{1}^{\|}=2 J_{1}^{\top} \frac{\left\langle S_{A}^{x}\right\rangle}{\left\langle S_{B}^{x}\right\rangle}\left\langle S_{B}^{z}\right\rangle .
\end{aligned}
$$

These equations are quite important and also hold in the classical mean-field approximation, where the state of the system is defined by these equations together with $\sqrt{\left\langle S_{A}^{x}\right\rangle^{2}+\left\langle S_{A}^{z}\right\rangle^{2}}=\frac{1}{2}$ and $\sqrt{\left\langle S_{B}^{x}\right\rangle^{2}+\left\langle S_{B}^{z}\right\rangle^{2}}=\frac{1}{2}$. Equation (15) also determines possible second order phase transitions as was shown my Matsuda and Tsuneto [15]. The normal fluid to superfluid (FE-CFE) second order transition is located at:

$$
h_{F E-C F E}^{z}=J_{1}^{\top}+J_{2}^{\top}-J_{1}^{\|}-J_{2}^{\|} .
$$

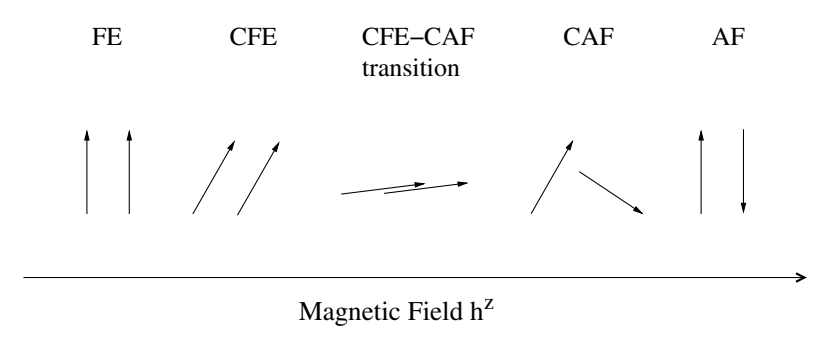

Fig. 2. The possible mean-field phases of the anisotropic Heisenberg model on a bipartite lattice with external field $h^{z}$.

For the normal solid to supersolid (CAF-AF) the classical mean-field approximation yields:

$$
h_{C A F-A F}^{z}=\sqrt{\left(-J_{1}^{\|}+J_{2}^{\|}-J_{2}^{\top}\right)^{2}-\left(J_{1}^{\top}\right)^{2}}
$$

and the critical magnetic field $h^{z}$ (corresponds to the chemical potential $\mu$ in the language of the QLG) for the superfluid to supersolid (CFE-CAF) transition is:

$$
\begin{aligned}
h_{C F E-C A F}^{z}= & \frac{J_{1}^{\|}+J_{2}^{\|}-J_{1}^{\top}-J_{2}^{\top}}{J_{1}^{\|}-J_{2}^{\|}-J_{1}^{\top}+J_{2}^{\top}} \\
& \times \sqrt{\left(-J_{1}^{\|}+J_{2}^{\|}-J_{2}^{\top}\right)^{2}-\left(J_{1}^{\top}\right)^{2}} .
\end{aligned}
$$

For a particular choice of coupling constants all four phases will exists when, see Figure 2:

$$
h_{F E-C F E}^{z}>h_{C F E-C A F}^{z}>h_{C A F-A F}^{z} .
$$

The three equations for the critical fields, equations (16)-(18), are derived for in classical mean-field approximation. However as equation (15) also holds in the random-phase approximation these values give a good indication where the actual transitions take place. Nevertheless, due to depletion of the spin-fields caused by quantum fluctuations the actual values are slightly lower, see Section 8.

\section{Thermodynamic properties}

In the first section we have seen that the grand-canonical QLG Hamiltonian, where the number of particles are variable corresponds to the canonical anisotropic Heisenberg Hamiltonian with fixed number of spins. Therefore there exists following relation between any thermodynamic potential defined in the QLG model and the anisotropic Heisenberg model:

$$
\Theta_{Q L G}-\mu N=\Theta_{\text {Heisenberg }}
$$

here $\Theta$ refers to an arbitrary thermodynamic potential. The self-consistency equations, derived in the previous sections, determine the spin fields of the anisotropic Heisenberg model in the various phases, but in regions of $h^{z}$ where more than one solution may exist, we have to compare internal energies to select the true ground 
state. Intuitively, we might want to compute the internal energy by calculating the expectation value of the Hamiltonian as:

$$
\begin{aligned}
H= & h^{z} \sum_{i}\left\langle S_{i}^{z}\right\rangle+\sum_{i j} J_{i j}^{\|}\left\langle S_{i}^{z} S_{j}^{z}\right\rangle \\
& +\sum_{i j} J_{i j}^{\top}\left(\left\langle S_{i}^{x} S_{j}^{x}\right\rangle+\left\langle S_{i}^{y} S_{j}^{y}\right\rangle\right)
\end{aligned}
$$

where the correlation functions can be derived from the corresponding Green's functions, equation (12). Unfortunately this approach will yield incorrect and inconsistent results as the Green's function derived with the cumulant decoupling is not an exact solution of the anisotropic Heisenberg Hamiltonian but rather the solutions of an unknown, effective model, which is an approximation of the Heisenberg model. As we do not know the exact form of this effective model we have to resort to fundamental thermodynamic relations to integrate the energy. At $T=0$ following equality holds:

$$
\frac{\partial U}{\partial h^{z}}=\frac{\left\langle S_{A}^{z}\right\rangle+\left\langle S_{B}^{z}\right\rangle}{2}
$$

This equation allows us to determine if the critical fields given by equations (16)-(18) really refer to second order phase transitions. As the $z$-component of the spin is decreased in the canted ferromagnetic phase due to the onset of the transversal field this phase is energetically favorable over the ferromagnetic phase. Similarly the canted anti-ferromagnetic phase has lower energy than the anti-ferromagnetic phase as the total magnetization in $z$-direction in the canted anti-ferromagnetic phase is somewhat higher due to the increasing influence of the ferromagnetic term $h^{z} \sum_{i} S_{i}^{z}$. Hence, the phases as depicted in Figure 2 are real.

Unfortunately equation (22) only allows one to determine the energetically favourable state when the possible transition point is known, such as in second order phase transitions. But we can not use the relation to determine possible first order transitions and unfortunately this shortcoming is only resolvable by extending the formalism to finite $\mathrm{T}$.

Thermodynamic relations connect various macroscopic quantities and we will use them to obtain observable properties. Although the external magnetic field $h^{z}$ in the spin model is an observable the corresponding quantity in the QLG model, namely the chemical potential is not. Therefore we are interested to attain a formula for the pressure associated with a certain chemical potential. The relationships is most easily derived from the following Maxwell relation:

$$
\left(\frac{\partial P}{\partial \mu}\right)_{T, V}=\left(\frac{\partial N}{\partial V}\right)_{T, \mu}=\frac{\#_{\text {lattice_sites }}}{V}(1-\epsilon)
$$

where $\epsilon:=\left\langle S_{A}^{z}\right\rangle+\left\langle S_{B}^{z}\right\rangle$. These are the basic thermodynamic properties that we will use in the further discussion and in principal all other properties can be derived from the internal energy $U$.

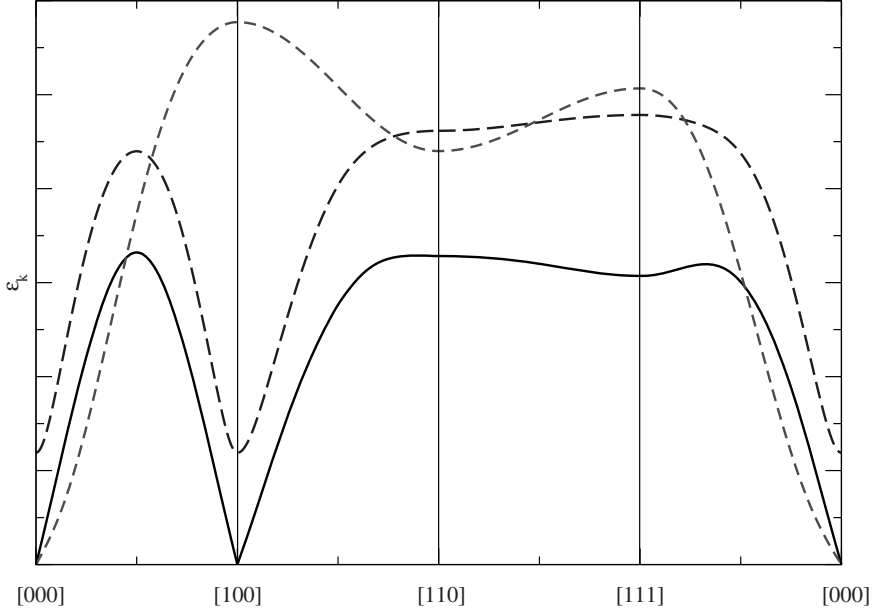

Fig. 3. Excitation spectrum of the anisotropic Heisenberg model with $J_{1}^{\top}=1.498 K, J_{2}^{\top}=0.562 K, J_{1}^{\|}=-3.899 K$ and $J_{2}^{\|}=-1.782 \mathrm{~K}$ for the supersolid phase (solid line and dashed line) at $h^{z}=0.65$ and the superfluid phase (long dashed line) at $h^{z}=7.46$. Here [000], [100], [110], [110] refer to the various points of the first Brillouin zone.

\section{Excitation spectrum}

The excitation spectrum in the ferromagnetic phase as well as in the anti-ferromagnetic phase feature the well known, gaped magnon excitation with quadratic $k$ dependence in the $k \rightarrow 0$ limit. In the canted ferromagnetic (superfluid) phase the spin-wave excitation spectrum is comprised, due to spontaneously broken $U(1)$ symmetry, of the gapless linear Goldstone phonons.

Additionally the canted anti-ferromagnetic (supersolid) phase exhibits a second branch which accounts for the broken translational symmetry. This second branch goes quadratic with $k$ in the long wavelength limit and has a gap:

$$
\begin{aligned}
\Delta= & {\left[J_{1}^{\top} 2\left(J_{1}^{\|}-J_{2}^{\|}+J_{2}^{\top}\right)\left\langle S_{A}^{x}\right\rangle^{2}\left\langle S_{B}^{x}\right\rangle^{2}\right.} \\
& +J_{1}^{\top 2}\left(\frac{\left\langle S_{A}^{x}\right\rangle^{2}+\left\langle S_{A}^{z}\right\rangle^{2}}{\left\langle S_{A}^{x}\right\rangle}\left\langle S_{B}^{x}\right\rangle^{3}+\frac{\left\langle S_{B}^{x}\right\rangle^{2}+\left\langle S_{B}^{z}\right\rangle^{2}}{\left\langle S_{B}^{x}\right\rangle}\left\langle S_{A}^{x}\right\rangle^{3}\right. \\
& \left.\left.+2\left\langle S_{A}^{x}\right\rangle\left\langle S_{B}^{x}\right\rangle\left\langle S_{A}^{z}\right\rangle\left\langle S_{B}^{z}\right\rangle\right)\right]^{\frac{1}{2}} .
\end{aligned}
$$

In Figure 3 the quasi-particle excitation spectrum is plotted for the superfluid (CFE) and the supersolid (CAF) phases. In the supersolid (CAF) phase the excitation energy reaches zero at the edge (point [100]) of the first Brillouin zone. The corresponding spin-waves refer to oscillations with a $\pi$-phase shift between different sublattices. Hence, on a single sub-lattice the spin-wave looks like a zero wave-number mode. It was recently suggested [11] that the superfluid to supersolid transition is triggered by a collapsing roton minimum. This assumption is supported by the present model; here the transition to the supersolid phase takes place when the excitation spectrum goes soft at [100]. The dispersion relation in the 
superfluid (CFE) phase is given by:

$$
\begin{aligned}
\omega(k)= & 2\left\{\left(J_{1}^{\top}\left(\gamma_{1}(k)-1\right)+J_{2}^{\top}\left(\gamma_{2}(k)-1\right)\right)\right. \\
& \times\left[\left\langle S_{z}\right\rangle^{2}\left(J_{1}^{\top}\left(\gamma_{1}(k)-1\right)+J_{2}^{\top}\left(\gamma_{2}(k)-1\right)\right)\right. \\
& \left.\left.-\left\langle S_{x}\right\rangle^{2}\left(J_{1}^{\top}+J_{2}^{\top}-J_{1}^{\|} \gamma_{1}(k)-J_{2}^{\|} \gamma_{2}(k)\right)\right]\right\}^{1 / 2},
\end{aligned}
$$

where $\gamma_{1}(k)$ and $\gamma_{2}(k)$ denote the standard lattice generating functions of a BCC lattice. From this equation we can see that the energy possibly goes to zero at [100] (corresponds to $\gamma_{1}(k)=-1$ and $\gamma_{2}(k)=1$ ) when the following condition is fulfilled:

$$
J_{1}^{\top}+J_{2}^{\top}+J_{1}^{\|}-J_{2}^{\|}<0 .
$$

Hence we obtained a further condition (supplementary to Eq. (18)) for the existence of the superfluid to supersolid transition.

Equation (25) allows for the existence of a second region of the reciprocal space where the dispersion relation might go soft. For $\gamma_{1}(k)=0$ and $\gamma_{2}(k)=-1$ which corresponds to [111] we obtain following condition:

$$
\frac{-2 J_{2}^{\top}}{J_{1}^{\top}+J_{2}^{\top}+J_{2}^{\|}}>0 .
$$

It was also conjectured that the superfluid phase in the vicinity of the superfluid to supersolid transition is unstable against spontaneously induced superflow and superflow associated with vortices. Therefore we investigate how the present model reacts to induced superflow. A net superflow is either given by a moving condensate which results in a gradient of the phase of the wave-function, or equivalently by a moving environment while the condensate stays at rest. The latter is obtained by an additional term in the Hamiltonian:

$$
H_{1}=\int d^{3} x \psi^{\dagger}(x)\left(i \mathbf{v}_{\mathbf{n}} \cdot \nabla\right) \psi(x)
$$

which corresponds to following term in the anisotropic Heisenberg model:

$$
H_{1}=\sum_{i j} J_{i j}^{\times}\left(S_{i}^{x} S_{j}^{y}-S_{j}^{x} S_{i}^{y}\right)
$$

here the nearest and next-nearest neighbor cross coupling constant are anti-symmetric $J_{i j}^{\times}=-J_{j i}^{\times}$and are zero for directions perpendicular to the motion of the environment $\mathbf{v}_{\mathbf{n}}$. The term yields an additional matrix $M_{1}$ in he random-phase approximation:

$$
M_{1}=\left(J_{1}^{\times}(k)+J_{2}^{\times}(k)\right)\left(\begin{array}{rrr}
\left\langle S^{z}\right\rangle & 0 & 0 \\
0 & \left\langle S^{z}\right\rangle & 0 \\
-\left\langle S^{x}\right\rangle & 0 & 0
\end{array}\right) .
$$

The matrix is reduced to dimension $3 \times 3$ because we are only interested in the superfluid phase only where no difference between the two sub-lattices is made. The cross coupling terms $J^{\times}$are given by:

$$
\begin{aligned}
J_{1}^{\times} & =\sum_{a_{A B}} J_{A B}^{\times} \exp \left(i k a_{A B}\right) \\
J_{1}^{\times} & =\sum_{a_{A A}} J_{A A}^{\times} \exp \left(i k a_{A A}\right),
\end{aligned}
$$

where $a_{A A}$ and $a_{A B}$ are the lattice parameters corresponding to $\mathrm{A}$ and $\mathrm{B}$ lattice sites. The dispersion relations, given by the eigenvalues of the total matrix $M+M_{1}$, are altered in the following way:

$$
\omega_{k} \rightarrow\left(J_{1}^{\times}(k)+J_{2}^{\times}(k)\right)+\omega_{k}
$$

in the $k \rightarrow 0$ limit this accounts for a tilt of the dispersion curve toward the motion of the environment; the quasi-particle energy in the direction of the motion $v_{n}$ is lowered while the energy for particles travelling in opposite directions is lifted. From the definition of the lattice generating functions $\gamma_{1}(k)$ and $\gamma_{2}(k)$ and equation (31) we see that $J_{1}^{\times}(k)=J_{2}^{\times}(k)=0$ for $\mathrm{k}$ where $\gamma_{1}(k)=-1$ and $\gamma_{2}(k)=1$. Hence the roton dip that triggers the superfluid to supersolid transition is not affected by the superflow. The situation is likewise for the roton minimum at [111] where $\gamma_{1}(k)=0$ and $\gamma_{2}(k)=-1$. Also here the cross couplings $J_{1}^{\times}$and $J_{2}^{\times}$become zero and the stability is not affected by induced superflow.

\section{Quantum fluctuations at zero temperature}

As presented in reference [13] quantum fluctuations are important to study as they can lead to the understanding of the physical origin of the different phases. At zero temperature there are no thermal fluctuations present in the system and all fluctuations will stem from quantum mechanical effects. The mean-field approximation as derived in the beginning of the paper is a classical approximation and as such it does not display quantum fluctuations. This is expressed by a constant spin magnitude of $1 / 2$ over all phases at zero temperature. In the anisotropic Heisenberg model quantum fluctuations are a result of non-vanishing pair correlations $\left\langle S_{i}^{\mu} S_{j}^{\nu}\right\rangle-\left\langle S_{i}^{\mu}\right\rangle\left\langle S_{j}^{\nu}\right\rangle$ of nearest and next nearest neighbors. Consequently, as randomphase approximation takes those correlations accurately into account we expect quantum fluctuations which are expressed in a depletion of the total spin magnitude as can be seen in Figure 4. In the ferromagnetic phase the total spin is $1 / 2$ and thus there are no quantum fluctuations present. This is expected as the ferromagnetic phase is governed in the $h^{z} \rightarrow \infty$ limit by an effective single operator, and hence the classical Hamiltonian:

$$
H=\sum_{i} h^{z} S_{i}^{z}
$$

The spin depletion is strongly pronounced in the CFE and $\mathrm{CAF}$ phases where transversal components account for additional fluctuations. We also see that in the CAF 


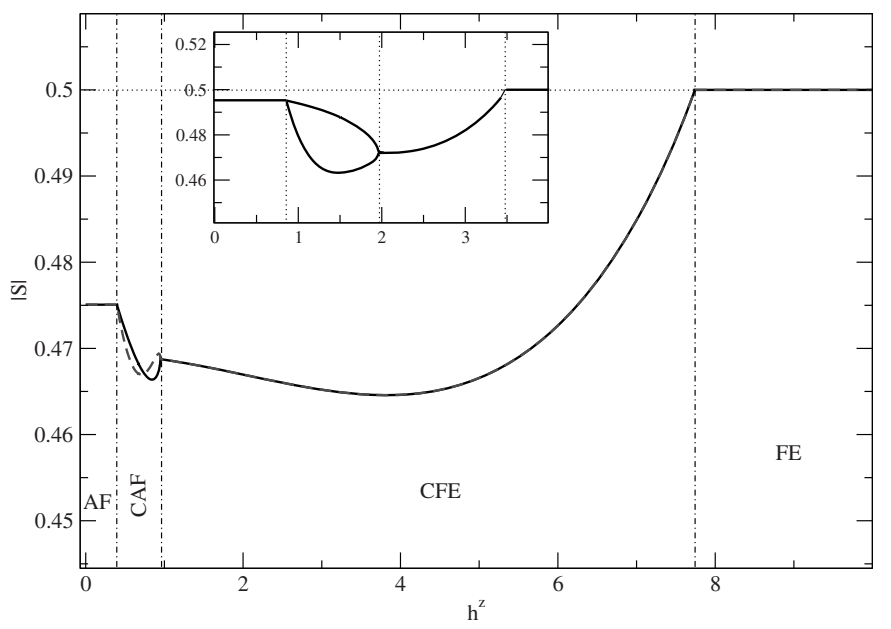

Fig. 4. The total spin magnitude at zero temperature is shown for all four phases, for the cases $J_{1}^{\top}=1.498 \mathrm{~K}, J_{2}^{\top}=0.562 \mathrm{~K}$, $J_{1}^{\|}=-3.899 K$ and $J_{2}^{\|}=-1.782 K$, main plot and $J_{1}^{\top}=0.5 K$, $J_{2}^{\top}=0.5 K, J_{1}^{\|}=-2 K$ and $J_{2}^{\|}=-0.5 K$, inset, respectively. The mean-field solution gives always the horizontal dotted line. In RPA the total spin is depleted due to quantum fluctuations. For the supersolid (CAF) phase the RPA gives a difference between the sublattices A and B (solid and dashed lines main plot). For details, see text.

phase the spin magnitude is different on the two sublattices. This indicates that next nearest neighbor interactions are dominant and balancing nearest neighbor integrations are slightly suppressed. Therefore we assume that in the canted anti-ferromagnetic phase the two sublattices do slightly decouple. This interpretation is supported by the spin-wave excitation spectrum in the canted anti-ferromagnetic phase. We have seen that there exists a zero frequency mode, where the spins on different sublattices are $\pi$-phase shifted. Hence this spin-wave which couples both sub-lattices carries no energy.

\section{Discussion}

\subsection{Case 1}

In this section we will discuss the properties of the supersolid phase using the example of two sets of coupling constants. As we are interested in describing real systems we may ask what sets of parameters are physical and which set exhibits the best fit to ${ }^{4} \mathrm{He}$. Physically, the transversal constants $J^{\top}$ ought to be positive as they correspond to the kinetic energy. In quantum lattice gas models usually $J^{\top} \mathrm{s}$ are chosen so that the kinetic energy is isotropic up to 4th order giving the best possible approximation to the continuum limit. However in the supersolid phase the Hamiltonian may be regarded as an effective model and therefore we refrain from this restriction. The interactions between the helium atoms are controlled by van-der-Waals forces and their repulsive nature at very short distances determines negative nearest neighbor interaction $J_{1}^{\|}$, evoking anti-ferromagnetic ordering in the spin language. The corresponding Lennard-Jones potential is short ranged and therefore it is sufficient to only consider nearest and next nearest neighbor interactions. Liu and Fisher chose coupling constants in order to fit the model to the actual phase diagram of Helium-4. As the supersolid phase had not been discovered experimentally at this time they investigated the possibility of a supersolid phase existing.

It is widely accepted that the lambda transitions falls into the universality class of the XY-model, which is a limiting case of the anisotropic Heisenberg model. In the same way we believe that the anisotropic Heisenberg model is capable of covering the essential properties of the supersolid phase. Nevertheless the present model will fail to appropriately map ${ }^{4} \mathrm{He}$ over the complete range of temperature and pressure as various properties such as variability of the lattice constant and lattice vibration modes (phonons) are not taken into account in this model. Therefore we abstain from fitting the solutions of randomphase approximation to the phase diagram of real ${ }^{4} \mathrm{He}$ but merely choose two sample sets to study key properties of the supersolid phase.

The first set of parameters is given by:

$$
\begin{aligned}
J_{1}^{\top} & =0.5 K \\
J_{2}^{\top} & =0.5 K \\
J_{1}^{\|} & =-2 K \\
J_{2}^{\|} & =-5 K .
\end{aligned}
$$

In the classical mean-field this set of parameters exhibits all four phases where the corresponding critical magnetic fields are given by equations (16)-(18). and yield respectively: $h_{F E-C F E}^{z}=3.5, h_{C F E-C A F}^{z}=2.0207$ and $h_{C A F-A F}^{z}=0.86608$. In the classical mean-field as well as in the random-phase approximation the transitions are determined by equation (15), which shows that $h^{z}$ roughly scales with $S_{A}^{z}$ and $S_{B}^{z}$. Therefore we expect that the transitions in the random-phase approximation due to depletion of the spin magnitude are slightly lower. The actual values are: $h_{F E-C F E}^{z}=3.5, h_{C F E-C A F}^{z}=1.96$ and $h_{C A F-A F}^{z}=0.857$.

The second set we have chosen was extensively studied by Liu and Fisher and their parameters are given by:

$$
\begin{array}{r}
J_{1}^{\top}=1.498 K \\
J_{2}^{\top}=0.562 K \\
J_{1}^{\|}=-3.899 K \\
J_{2}^{\|}=-1.782 K .
\end{array}
$$

Again the transition points are slightly lower (except for the FE-CFE transition) for the random-phase approximation and yield: $h_{F-C F E}^{z}=7.741(7.741), h_{C F E-C A F}^{z}=$ $1.0071(1.0577)$ and $h_{C A F-A F}^{z}=0.3963(0.41716)$, where the numbers in parentheses are the values derived by the classical mean-field approximation. Figure 5a depicts the relation between the external field of the anisotropic Heisenberg model and the pressure in the QLG as given by 

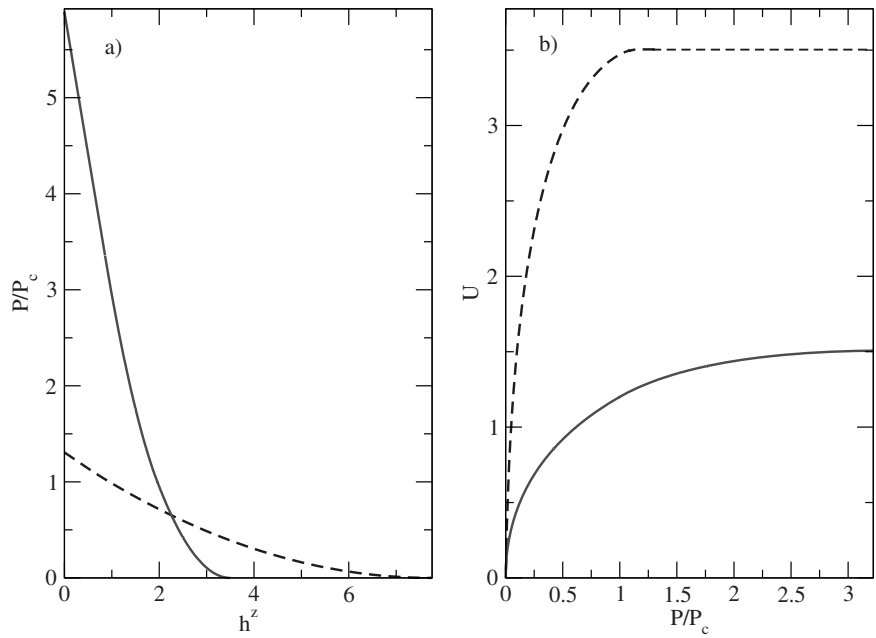

Fig. 5. (a) shows the relation between pressure in the QLG model and the external magnetic field in the anisotropic Heisenberg model. The pressure is normalized with respect to the critical pressure $P_{c}$ marking the supersolid to superfluid transition. The dashed line refers to set $1: J_{1}^{\top}=1.498 \mathrm{~K}$, $J_{2}^{\top}=0.562 K, J_{1}^{\|}=-3.899 K$ and $J_{2}^{\|}=-1.782 K$ and the solid line to set 2 : $J_{1}^{\top}=0.5 K, J_{2}^{\top}=0.5 K, J_{1}^{\|}=-2.0 K$ and $J_{2}^{\|}=-0.5 K(\mathrm{~b})$ depicts the internal energy of the model as a function of the pressure for set 1 (dashed line) and set 2 (solid line).

equation (23). The pressure on the $y$-axis is renormalised so that 1 corresponds to the critical pressure $P_{c}$ of the superfluid to supersolid transition, given by roughly 20 atm in Helium-4. High magnetic field corresponds to low pressure and vice versa. Negative magnetic fields correspond to high pressures that do not have physical validity in the quantum lattice gas. Therefore the maximal pressure corresponds to zero magnetic fields.

In Figure 5b we plotted the internal energy of the anisotropic Heisenberg model which corresponds to the conjugated potential $U[\mu]=U-\mu N$ per volume of the QLG model which is minimized at zero temperature. In Figure 6 we plotted the superfluid order parameter $\langle\psi\rangle$ as a function of the pressure in the superfluid and supersolid phase for both sets of parameters. The order parameter displays its maximum value in the vicinity of the transition to the superfluid phase and evidently approaches zero at the NS-SS transition. The superfluid order parameter on the on-site sub-lattice associated with vacancies is higher than the one on the interstitial sub-lattice. While this effect is strongly pronounced in Set 1 , where the order parameter of the vacancies is around 37 times the order parameter of the interstitials near the SS-NS transition, in Set 2 the Bose condensation of the vacancies is only marginally higher (1.3 times) than of the interstitials. Yet we observe that in this model Bose condensation appears in the vacancies as well as in the interstitials though the major contribution comes from the vacancies. In Figure 7 we have the density of vacancies, the interstitials and the difference of both, the net vacancy density plotted as a function of the pressure in the supersolid and normal solid
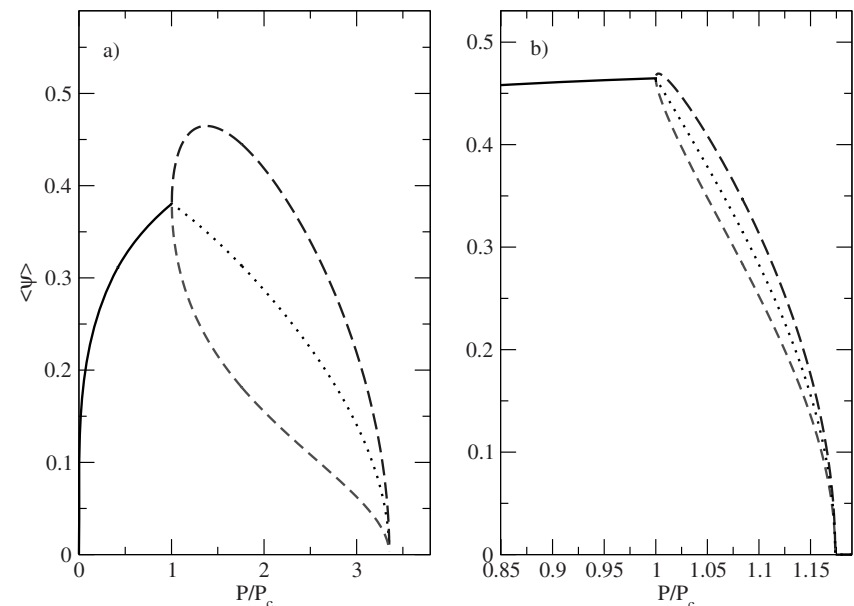

Fig. 6. Magnitude of the superfluid order parameter $\langle\psi\rangle$ in the supersolid and superfluid phase. Long dashed line refers to the on-site sub-lattice A while the dashed line refers to the interstitial sub-lattice B. The dotted line is the average of both. (a) refers to set 1 and (b) to set 2 (see text).

phases. We see that in the normal solid phase the number of vacancies and interstitials stays finite. This is due to quantum fluctuations and consequently the number of vacancies have in addition to thermal activation a second contribution resulting from quantum mechanical effects. Nevertheless the number of vacancies and interstitials appear in equal numbers and the net contribution in the normal solid is zero. This is different in the supersolid phase where a surplus of vacancies accounts for a positive net vacancy density. As we decrease the pressure in the supersolid phase both the vacancy density and the interstitial density increase. However, the vacancy density increases faster, leaving a net vacancy density which reaches its maximum at the phases transition between the supersolid and the superfluid. Interestingly the net vacancy density varies nearly linearly with the pressure as the solid line in Figure 7 shows.

\subsection{Case 2}

In the section on the excitation spectrum we have seen that the spin-wave energy at [100] of the first Brillouin zone goes soft exactly when the superfluity to supersolid phase transition occurs. Additionally, for coupling constants that fulfill condition equation (27) there exists a second minimum at [111] which can collapses. Following set of constants fulfill this condition:

$$
\begin{aligned}
J_{1}^{\top} & =0.5 \mathrm{~K} \\
J_{2}^{\top} & =0.5 \mathrm{~K} \\
J_{1}^{\|} & =-2 \mathrm{~K} \\
J_{2}^{\|} & =-1.5 \mathrm{~K} .
\end{aligned}
$$

According to equation (16) there is one (normal fluid to superfluid) transition in the system:

$$
h_{F E-C F E}^{z}=4.5
$$



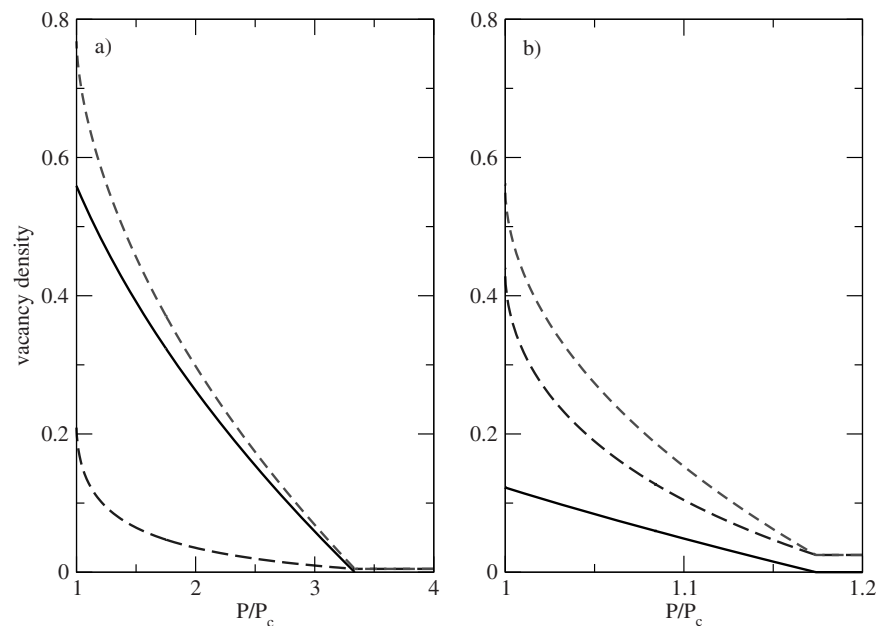

Fig. 7. Density of vacancies (dashed line), density of interstitials (long dashed line) and the difference of both, the net vacancy density (solid line) in the normal solid (for $P / P_{c}>1$ ) and the supersolid phases (below 1). (a) refers to set 1 and (b) to set 2 (see text).

beneath this line classical mean-field approximation predicts a CFE (superfluid) phase that extends down to $h^{z}=0$ as due to the relatively large negative $J_{2}^{\|}$the solid phase does not acquire a sufficiently low free energy to be the true ground state. The random-phase approximation however draws a slightly different picture. Analogous to the classical mean-field solution the random-phase approximation also yields a phase transition near $h^{z}=4.5$. But unlike the classical mean-field solution, the superfluid phase here does not survive all the way down to $h^{z}=0$. Due to the particular choice of parameters the superfluid phase becomes unstable at around $h^{z}=2$; i.e the quasi-particle spectrum turns imaginary at $\gamma_{1}(k)=0$ and $\gamma_{2}(k)=-1$ ([111]), as Figure 8 shows. The dashed line in this figure shows the excitation spectrum under an induced superflow $v_{s}$. The roton minimum is not affected by this superflow and hence the superfluid phase is not destabilised by an spontaneously induced superflow. Interestingly beyond this line no other stable phase exists in the random-phase approximation; there is no set of spin fields $\left\langle S_{A}^{x}\right\rangle,\left\langle S_{B}^{x}\right\rangle,\left\langle S_{A}^{z}\right\rangle$ and $\left\langle S_{B}^{z}\right\rangle$ that solves the self-consistency equations of the random-phase approximation. Therefore we conclude that there must exist a 'novel' phase that is not covered by the random-phase approximation on a bipartite lattice and we will leave the detailed discussion of this phase to future work.

\section{Conclusion}

In this paper we analysed the supersolid phase in the three dimensional quantum lattice gas model. Through transformation to the anisotropic Heisenberg model in a external field we were able to employ the well-established technique of real-time Green's functions for spin systems. The series of infinite order Green's functions as it appears in

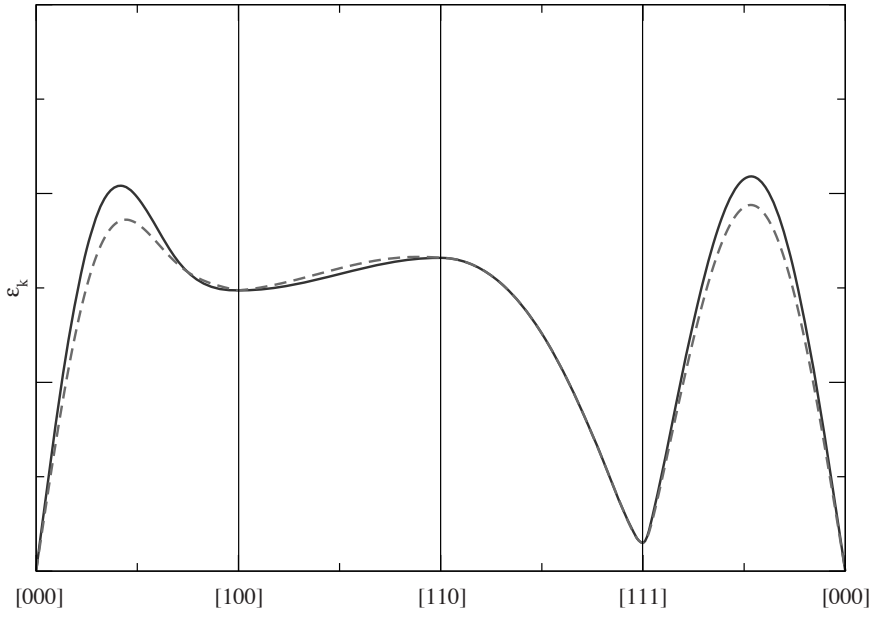

Fig. 8. Excitation spectrum (solid line) in the superfluid phase for $J_{1}^{\top}=0.5 K, J_{2}^{\top}=0.5 K, J_{1}^{\|}=-2 K J_{2}^{\|}=-1.5 K$ just before the phase become unstable due to a collapsing minimum at [111]. Induced superflow alters the spectrum (dashed line) but does not affect the minimum.

the equation of motion was truncated by applying cumulant decoupling and the resulting random-phase approximation accounts for linear spin-waves. We are the first to apply this method to the canted anti-ferromagnetic phase entailing a set of 6 algebraic equations. The innate selfconsistency equations inhere a 3 dimensional numerical integral over the $k$-space. By introducing a two dimensional density of states the integral was reduced to two dimensions where the lattice generating functions serve as new integration variables. In the said integral the DOS is the only quantity that depends on the structure of the lattice. Hence, once the DOS is computed for a certain lattice geometry the further calculation remain unaltered. Therefore our method is widely applicable and easily adjustable to various magnetic systems where canted phases are in the center of interest. This also holds for 2 dimensional lattices where linear spin waves are expected to yield a reasonable approximation.

The random-phase approximation takes quantum fluctuations into account and consequently in this solution the solid phase exhibits vacancies and interstitials at zero temperature. Yet in the normal solid phase the vacancies and the interstitials occur in equal number, thus yielding a zero net vacancy density. In the supersolid phase this balance shifts in favour of the vacancies giving rise to a finite positive net vacancy density at zero temperature. Our data also shows that vacancies as well as interstitials Bose condense and hence both contribute to superfluidity. Nevertheless the Bose condensation is stronger expressed in the vacancies thus giving the major contribution to supersolidity.

Furthermore the present approach confirms suggestions that the superfluid to supersolid transition is triggered by a collapsing roton minimum. However our results show that this roton dip is not affected by Galilean transformation and hence the superfluid phase is stable against spontaneously induced superflow. Additionally we 
find that for a narrow regime of parameters a second roton minimum collapses. Beyond this point does not exist a stable phase in the bipartite random-phase approximation and is thus beyond the model. The prospect of future work looks promising. The formalism is easily extendable to finite temperatures as shown in reference [22], where we investigated the properties of the supersolid phase at finite T. In particular the temperature dependence of the net vacancy density and the behavior of the specific heat across the supersolid to normal solid transitions is of particular interest.

\section{References}

1. A.F. Andreev, I.M. Lifshitz, Zh. Eksp. Teor. Fiz. 56, 2057 (1969) [JETP 29, 1107 (1969)]

2. G.V. Chester, Phys. Rev. A 2, 256 (1970)

3. A.J. Leggett, Phys. Rev. Lett. 25, 1543 (1970)

4. E. Kim, M.H.W. Chan, Nature 427, 225 (2004)

5. E. Kim, M.H,W. Chan, Science 305, 1941 (2004)

6. Y. Aoki, J.C. Graves, H. Kojima, Phys. Rev. Lett. 99, 015301 (2007)
7. J. Day, J.R. Beamish, J. Nature 450, 853856 (2007)

8. P.W. Anderson, Nature Phys. 3, 160 (2007)

9. M. Boninsegni, N. Prokof'ev, B. Svistunov, Phys. Rev. Lett. 96, 105310 (2006)

10. K.-S. Liu, M.E. Fisher, J. Low. Temp. Phys. 10, 655 (1973)

11. P. Nozières, J. Low Temp. Phys. 137, 45 (2004)

12. E. Zhao, A. Paramekanti, Phys. Rev. Lett. 96, 105303 (2006)

13. A. Stoffel, M. Gulacsi, Europhys. Lett, in print

14. T. Matsubara, H. Matsuda, Progr. Theoret. Phys. 16, 569 (1956); T. Matsubara, H. Matsuda, Progr. Theoret. Phys. 17, 19 (1957)

15. H. Matsuda, T. Tsuneto, Prog. Theoret. Phys. Suppl. 46, $411(1970)$

16. M.E. Fisher, Rep. Prog. Phys. 30, 615 (1967)

17. N.N. Bogolyubov, S.V. Tyablikov, Doklady Akad. Nauk. S.S.S.R. 126, 53 (1959) [translation: Soviet Phys. -Doklady 4, 604 (1959)]

18. S.V. Tyablikov, Ukrain. Mat. Yhur. 11, 287 (1959)

19. A. Auerbach, Interacting electrons and quantum magnetism (Springer, 1994)

20. P.E. Bloomfield, E.B. Brown, Phys. Rev. B 22, 1353 (1980)

21. P.E. Bloomfield, N. Nafari, Phys. Rev. A 5, 806 (1972)

22. A. Stoffel, M. Gulacsi, Eur. Phys. J. B, in print 